\title{
Low temperature variation of the dielectric constant of the amorphous glycerol at $9.15 \mathrm{GHz}$
}

\author{
A. Bachellerie and P. Legros \\ Laboratoire d'Ultrasons (*), Université Pierre-et-Marie-Curie, Tour 13, 4, place Jussieu, 75230 Paris Cedex 05, France
}

(Reçu le 21 avril 1980, réviséle 29 septembre, acceptéle 20 octohre 1980)

\begin{abstract}
Résumé. - La variation de la constante diélectrique du glycérol amorphe a été mesurée en fonction de la température. Une variation logarithmique en fonction de $T$ est observée entre 0,5 et 3,6 K. Ceci montre l'existence de systèmes à deux niveaux dans le glycérol avec une densité d'états presque constante pour des énergies inférieures à $4 \mathrm{~K}$. Le coefficient du logarithme népérien est égal à $2,3 \times 10^{-4}$.
\end{abstract}

\begin{abstract}
The variation of the dielectric constant of amorphous glycerol was measured as a function of the temperature. A logarithmic variation of $\Delta \varepsilon / \varepsilon$ versus $T$ is observed between $0.5 \mathrm{~K}$ and $3.6 \mathrm{~K}$. This gives some evidence for the existence of the two level systems in glycerol with a nearly constant density of states for energies smaller than $4 \mathrm{~K}$. The coefficient of the logarithm is equal to $2.3 \times 10^{-4}$.
\end{abstract}

1. Introduction. - Over the past few years considerable effort has been devoted to the study of amorphous materials. Their heat capacity, thermal conductivity [1] and acoustical properties [2] have been studied. Ultrasound wave (U.S.W.) propagation measurements are particularly appropriate for the study of amorphous materials. They allow one to choose between the different models that have been proposed to explain the properties of these materials [2]. A good many of the properties are qualitatively and, to a good accuracy, quantitatively explained in terms of the existence of localized excitations in form of two level systems (T.L.S.'s) with an approximately constant density of states [3].

Recently interaction between T.L.S. and electromagnetic waves (E.M.W.'s) has been discovered in amorphous materials doped with $\mathrm{OH}^{-}$dipoles [4]. The advantages of E.M.W.'s over U.S.W.'s lie in the relative simplicity of the E.M.W. experiments, and the fact that one can work at higher temperatures and frequencies with E.M.W.'s. One can thus expect to obtain more accurate measurements for the E.M.W. absorption in amorphous samples than in a U.S.W. experiment.

We have chosen to study glycerol because of its organic origin. Until now very few organic amor-

$\left(^{*}\right)$ Associated with the Centre National de la Recherche Scientifique. phous materials have been studied with E.M.W.'s. To our knowledge, only P.M.M.A., P.E.T. [5] and teflon [5-6] have been examined. All are polymers and all have a small variation of $\Delta \varepsilon / \varepsilon$ (very small for pure P.M.M.A. and of the order of the experimental accuracy for teflon). Moreover glycerol is also a trihydroxyalcohol. Taking into account this great number of dipoles, one may think of it as a dipolar glass [7].

Another interesting feature of glycerol is its facility for becoming amorphous. The amorphisation procedure is well known and is easy to implement. The physical properties of amorphous glycerol have been studied at high temperature $(T>90 \mathrm{~K})$ specifically, the dielectric constant and the influence of the pressure on the dielectric constant [8]. The molecular vibration and the Raman spectra have also been studied [9-10]. At low temperatures the heat capacity in both the crystalline and the glassy states has been measured versus the temperature and the rate of cooling [11] down to $2.3 \mathrm{~K}$.

In this article we report the variation of the dielectric constant of glycerol versus the temperature between 0.05 and $3.6 \mathrm{~K}$. We observe a logarithmic variation in the dielectric constant versus the temperature. In the whole temperature range observed, the variation of the dielectric constant is in agreement with a theoretical prediction based on the hypothesis of the existence of T.L.S. with a constant density of states. The coupling constant defined as 
the coefficient of the logarithm has been measured; it is equal to $2.3 \times 10^{-4}$. This is of the order of the constant measured on silica samples with a low density of $\mathrm{OH}^{-}$dipoles (1.5 ppm, see [4]).

2. Sample and experimental technique. - For this experiment we used the glycerol of Merck Company with the quality for analysis, containing less than $1 \mathrm{ppm}$ of heavy metals and $1 \mathrm{ppm}$ of iron, and approximately $13 \%$ water. The experiments were performed with a niobium cylindrical cavity $[6,12]$ filled with this glycerol. As is well known, superconductors are poor heat conductors at low temperatures. Consequently the niobium cavity was set inside an O.F.H.C. copper block. In order to obtain a good thermal contact, a vacuum grease was set between the two parts of the system and the copper block was then tightly fixed, using screws, on the niobium cavity. A hole was drilled on the upper part of the cavity. The glycerol inside the cavity was in thermal contact through the hole with the upper part of the copper block which was itself tightened with the lower part of the mixing chamber of the dilution cryostat. The cavity resonance frequency was obtained by measuring the frequency of the microwave transmitted power through the cavity with a high precision frequency meter (its relative precision being better than one part in $\left.10^{-8}\right)$. The maximum error on $\Delta \varepsilon / \varepsilon$ is $2.5 \times 10^{-5}$. The low temperature measurements were done with two speer resistances : one was placed on the upper part of the cavity between the cavity and the mixing chamber, the other on the lower part of the cavity which was connected to the mixing chamber by the copper block. Below $2.5 \mathrm{~K}$ the temperature was varied very slowly and the total duration of the experiments was about 5 hours from $2.5 \mathrm{~K}$ to $0.05 \mathrm{~K}$.

3. Experimental results. - We studied the variation of $\Delta \varepsilon / \varepsilon$ at $9.150 \mathrm{GHz}$ versus the temperature in the range $0.05 \mathrm{~K}$ to $3.6 \mathrm{~K}$. This variation versus $\log T$ is given in figure 1 (for clarity, only about one point out of five measured is reported). It can be seen that $\Delta \varepsilon / \varepsilon$ decreases logarithmically between $0.5 \mathrm{~K}$ and $3.6 \mathrm{~K}$. The total variation is of the order of $5 \times 10^{-4}$ which is approximately the same variation as for Suprasil I [4] in the same temperature range, but is 4 times lower than what has been observed for BK 7. Under $0.4 \mathrm{~K} \Delta \varepsilon / \varepsilon$ passes through a maximum at about $0.2 \mathrm{~K}$ and decreases slightly when the temperature decreases down to $0.05 \mathrm{~K}$.

4. Interpretation of the results. - We assume the existence of two level systems (T.L.S.) with energy $E=\hbar \omega_{\mathrm{r}}$ spreading from 0 to $\hbar \omega_{\mathrm{M}}\left(\omega_{\mathrm{M}}\right.$ is the angular frequency corresponding roughly to the energy of the melting point of the material $\hbar \omega_{\mathrm{M}}=k T_{\mathrm{M}}$ ). To perform the calculation of the variation of the dielectric constant we use the same method as for the

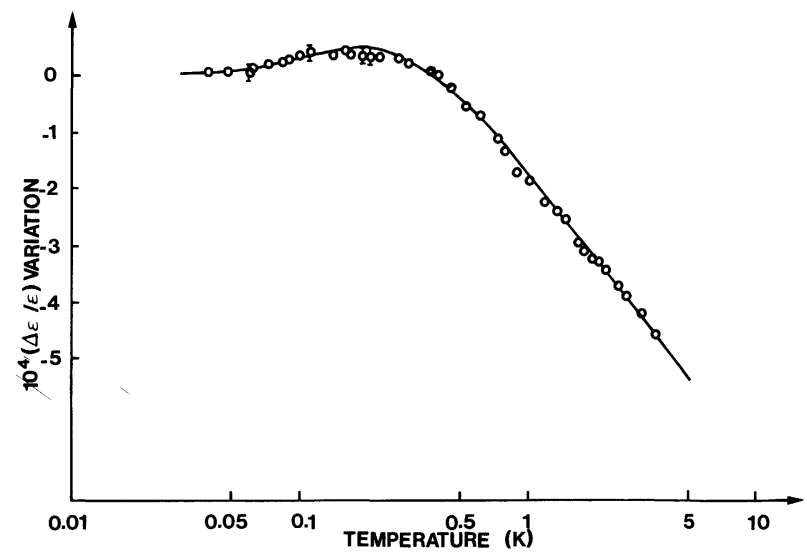

Fig. 1. - Semi-log plot of the linear variation of $\Delta \varepsilon / \varepsilon$ versus the logarithm of the temperature at a frequency of $9.15 \mathrm{GHz}$. The circles represent the experimental points (only about one out of five really measured points is reported). Estimated error bars of the measurements are shown. The continuous line represents the theoretical variation of $\Delta \varepsilon / \varepsilon$ deduced from equation (2).

elastic constant [14-15]. The Hamiltonian of the system is :

$$
\begin{aligned}
& H=H_{0}+H^{\prime} \\
& H_{0}=\frac{1}{2}\left|\begin{array}{cc}
E & 0 \\
0 & -E
\end{array}\right| \quad H^{\prime}=\frac{1}{2}\left|\begin{array}{cc}
\mu & +\mu^{\prime} \\
\mu^{\prime} & -\mu
\end{array}\right| F(t)
\end{aligned}
$$

$H_{0}$ refers to the unperturbated system, $H^{\prime}$ describes the coupling of the T.L.S. with a time dependent electric field $F(t)$ at frequency $\omega . H_{0}$ and $H^{\prime}$ are written in a basis in which $H_{0}$ is diagonal. The two unperturbated eigenstates have energies equal to $\pm E / 2$. The off-diagonal terms of $H^{\prime}$ represent a perturbation which induces transitions between the two eigenstates. They are of central importance in the following calculations of the dielectric constant change $\delta \varepsilon_{\text {res }}$ due to resonant effects.

In equation (1) $\mu$ and $\mu^{\prime}$ are respectively the dipole and induced dipole moments. One finds :

$$
\begin{aligned}
\delta \varepsilon_{\mathrm{res}} & =A \int_{0}^{\omega_{\mathrm{M}}} n\left(\hbar \omega_{\mathrm{r}}\right) \tanh \left(\frac{\hbar \omega_{\mathrm{r}}}{2 k T}\right) \times \\
& \times\left(\frac{\left(\omega_{\mathrm{r}}-\omega\right)}{\left(\omega_{\mathrm{r}}-\omega\right)^{2}+\Gamma^{2}}+\frac{\left(\omega_{\mathrm{r}}+\omega\right)}{\left(\omega_{\mathrm{r}}+\omega\right)^{2}+\Gamma^{2}}\right) \mathrm{d}\left(\hbar \omega_{\mathrm{r}}\right)
\end{aligned}
$$

with $\Gamma=T_{2}^{-1}$ where $T_{2}$ is the equivalent of the spinspin relaxation time and $A=\mu^{\prime 2}$.

The physical meaning of equation (2) is as follows. The function $\left(\omega_{\mathrm{r}}-\omega\right) /\left[\left(\omega_{\mathrm{r}}-\omega\right)^{2}+\Gamma^{2}\right]$ represents the anomalous dispersion of the E.M.W.'s due to the interaction with the T.L.S. of eigenfrequency $\omega_{r}$. The total dispersion is obtained by summing over all the T.L.S.'s, taking into account the density of states $n\left(\hbar \omega_{\mathrm{r}}\right)$ and the thermal population factor $\tanh \left(\hbar \omega_{\mathrm{r}} / 2 k T\right)$. It is generally assumed that the 
density of states of the T.L.S. is energy independent; there we adopt this hypothesis and we write $n\left(\hbar \omega_{\mathrm{r}}\right)=n_{0}$ where $n_{0}$ is a constant. Then the integral is calculated. The result is complicated. It can be simplified by using the conditions $\omega_{\mathrm{M}} \gg 2 k T / \hbar$, $\omega_{\mathrm{M}} \gg \omega, \omega_{\mathrm{M}} \gg \Gamma$ (always satisfied) and $\omega \gg \Gamma$ (true for our frequency [17]). According to which of the two conditions $2 k T>\hbar \omega$ or $2 k T<\hbar \omega$ is fulfilled, the two following regimes are found :

$$
\begin{aligned}
& \frac{\Delta \varepsilon}{\varepsilon}=-B \log \left(\frac{T}{T_{0}}\right) \text { for } 2 k T \gg \hbar \omega \\
& \frac{\Delta \varepsilon}{\varepsilon}=-B \log \left(\frac{\omega}{\omega_{\mathrm{M}}}\right) \text { for } 2 k T \ll \hbar \omega .
\end{aligned}
$$

The factor $B$ is equal to $2 n_{0} \mu^{\prime 2} / \varepsilon$ [18].

A maximum is expected for $T_{\mathrm{m}} \simeq \hbar \omega / 2.2 k$ [14]. In our case, with $\omega=2 \pi \times 9.15 \times 10^{9} \mathrm{rad}$./s, one must have $T_{\mathrm{m}} \simeq 0.2 \mathrm{~K}$. This is what was actually observed. In order to have a better look of the dielectric constant variation we have performed the integration in equation (2) with a computer. The numerical values of $B$ and $\Gamma$ are needed for input. If $\Gamma \ll \omega$ the result does not depend on $\Gamma$ (we have checked this point). Using for $B$ the value $2.3 \times 10^{-4}$, it is possible to superpose the theoretical variation with the experimental one. Between 0.5 and $3.6 \mathrm{~K}$ the variation of the dielectric constant is logarithmic, and such a result is generally considered a test of the existence of T.L.S. with an approximately constant density of states. In order to evaluate the accuracy of this test we can calculate the change $\delta \varepsilon_{\text {res }}$ when the density of states is not constant. For that purpose we choose arbitrarily the following form :

$$
n\left(\hbar \omega_{\mathrm{r}}\right)=n_{0}\left(1+\frac{\omega_{\mathrm{r}}}{\omega_{\mathrm{d}}}\right)
$$

where $\omega_{\mathrm{d}}$ is the angular frequency which scales the deviation of $n\left(\hbar \omega_{\mathrm{r}}\right)$ from a constant value. For $\hbar \omega \ll k T$, we find

$$
\frac{\Delta \varepsilon}{\varepsilon}=-B\left(\log \frac{T}{T_{0}}+\frac{k T}{\hbar \omega_{\mathrm{d}}}\right) .
$$

The deviation from a logarithmic law is smaller than $5 \%$ in the explored temperature range. Therefore we are able to conclude that the density of states is constant within an accuracy of $5 \%$ at least for T.L.S.'s whose energy is lower than $4 \mathrm{~K}$ (or frequency lower than $10^{11} \mathrm{~Hz}$ ).

These dielectric constant measurements provide us with a value of the product $n_{0} \mu^{\prime 2}$. In order to separate each of the two factors, another measurement is needed. In principle, specific heat measurements give the value of $n_{0}$. Up to now such an experiment has been done for temperatures higher than $2.3 \mathrm{~K} \mathrm{[11]}$; this is not sufficient for extracting a value for $n_{0}$. Moreover, a recent experiment [19] has shown that two kinds of T.L.S.'s may exist and contribute to the dielectric properties. Specific heat measurements are unable to distinguish between these two types of T.L.S. Therefore we will not enter further in this difficult question and we limit ourselves to the result : $B=2 n_{0} \mu^{\prime 2} / \varepsilon=2.3 \times 10^{-4}$.

Up to this point we have completely neglected the $\Delta \varepsilon / \varepsilon$ variation due to relaxational effects. These effects would give an increase of $\varepsilon(T)$ when $T$ increases and generally are only significant for temperatures higher than $5 \mathrm{~K}$ for our frequency. Our experiment confirms that they are negligible for $T \lesssim 4 \mathrm{~K}$.

5. Conclusion. - In this article it is shown that the resonant dispersion of electromagnetic wave due to T.L.S. also exists in glycerol. We observe a logarithmic variation of $\Delta \varepsilon / \varepsilon$ versus the temperature in the temperature range $0.5-4 \mathrm{~K}$. This behaviour may be explained with the T.L.S. model taking into account a constant density of states, at least for energies up to $4 \mathrm{~K}$. The agreement between the experiments and the theory is satisfactory in the whole temperature range explored $(0.05-4 \mathrm{~K})$. We show, for the first time, that the T.L.S., with a nearly constant density of states, exists in amorphous glycerol in spite of its organic, dipolar glass, and molecular character.

\section{References}

[1] Krüger, J., Phys. Chem. Glasses 13 (1972) 9 ; Stephens, R. B., Phys. Rev. B 8 (1973) 2896 ;

Lasjaunias, J. C., Ravex, A., Vandorpe, M., Hunklinger, B., Solid State Commun. 17 (1975) 1045.

[2] Golding, B., Grabner, J. E., Halperin, B. I. and Schutz, R. J., Phys. Rev. Lett. 30 (1973) 223 ;

Hunklinger, S., Proc. Ultrasonic Symp. (IEEE) (1974) 443 ; Arnold, W., Hunklinger, S., Stein, S. and Dransfeld, K., J. Non-Cryst. Solids 14 (1974) 192.

[3] Philips, W. A., J. Low Temp. Phys. 7 (1972) 351 ;

Anderson, P. W., Halperin, B. I. and Verma, C. M., Philos. Mag. 25 (1972).

[4] Von Schikfus, M., Hunklinger, S. and Piche, L., Phys. Rev. Lett. 15 (1975) 876;

Von Schikfus, M. and Hunklinger, S., J. Phys. C 9 (1976) L-439; Phys. Lett. 64A (1977) 144
[5] Von Schikfus, M., Laermans, C., Arnold, W. and HunkLINGER, S., Proc. 4th Int. Conf. "The physics of noncrystalline solids » (1976).

[6] MeYer, W., I.E.E. Trans. Microwave Theory Tech. 25 (1977) 1092.

[7] Lines, M. E., Phys. Rev. B 15 (1977) 388.

[8] Gildchrist, A., Earley, J. E. and Cole, R. H., J. Chem. Phys. 26 (1957) 196 ;

Scaife, W. G. S., J. Phys. D (Appl. Phys.) 9 (1976) 1489.

[9] Champeney, D. C. and Dean, G. W., J. Phys. C (Solid State Phys.) 8 (1975) 1276.

[10] Beaudoin, J. L., Manisse-Morgant, A., Benchename, A. and Harrand, M., C. R. Hebd. Séan. Acad. Sci. Paris 273 (1971) 15.

[11] Simon and Lange, Z. Phys. 38 (1926) 227 ; 
Ahlberg, J. E., Blanchard, E. R. and Lundberg, W. O., J. Chem. Phys. 5 (1937) 539;

Calemzuk, R., Lagnier, R. and Boujour, E., J. Non-Cryst. Solids 34 (1979) 149.

[12] Nguyen Tuong Viet, Ann. Phys. 2 (1967) 225.

[13] Kim, M. G., J. Chem. Soc. Faraday Trans. II 71 (1975) 415.

[14] Hunklinger, S. and Arnold, W., in Physical Acoustics, edited by R. N. Thurston and W. P. Mason (Academic Press, New York) 1976, Vol. 12, p. 155.

[15] Joffrin, J. and Levelut, A., J. Physique 36 (1975) 811.

[16] The form used here for $H^{\prime}$ is different from this used in [14] but with the choice done here we obtain a more symmetrical form for $E$ and $\delta P$. Evidently the coefficient for the variation of $\Delta \varepsilon / \varepsilon$ due to the resonant process is divided by four but not that of the relaxation (which is only related to $\mu$ ).
[17] Bachellerie, A., Doussineau, P., Levelut, A. and TA ThuThuy, J. Physique 38 (1977) 69 ;

Arnold, W. and Hunklinger, S., Solid State Commun. 17 (1975) 883

The $T_{2}$ was also measured at low temperature $(T<0.1 \mathrm{~K})$ : Golding, B. and Graebner, J. E., Phys. Rev. Lett. 37 (1976) 852 ;

Bernard, L., Piche, L., Schumacher, G. and Joffrin, J., J. Low Temp. Phys. 35 (1979) 411.

[18] $B$ is evidently a factor without dimension but it can be written as a function of quantities which are expressed in one system of units. In this article we have chosen the M.K.S.A system of units. The result takes also into account the choice of $\mu^{\prime}$ done in formula (1).

[19] Golding, B., Von Schikfus, M., Hunklinger, S. and DransFELD, K., Phys. Rev. Lett. 42 (1979) 1817. 